\title{
RNA Binding
}

National Cancer Institute

\section{Source}

National Cancer Institute. RNA Binding. NCI Thesaurus. Code C20737.

RNA Binding is the molecular interaction between an RNA molecule and a macromolecule (usually protein or nucleic acid) for transport, catalysis, localization, or modification of function. 